\title{
A Photogenerated Silicon Plasma Waveguide Switch and Variable Attenuator for Millimeter-Wave Applications
}

\author{
Thomas R. Jones, Member, IEEE, Alden Fisher, Graduate Student Member, IEEE \\ Douglas W. Barlage, Senior Member, IEEE and Dimitrios Peroulis, Fellow, IEEE
}

\begin{abstract}
This paper reports the design, fabrication, and measurement of a millimeter-wave solid-state $\pi$-match waveguide switch using bulk silicon micromachining. A photogenerated plasma within a silicon post is utilized as the switching element within the waveguide channel. Not only does this isolate the switch bias network from the RF signal path, but allows for tuning of the OFF-state isolation with increasing optical power for application as a variable attenuator. A measured OFF-state isolation greater than $25 \mathrm{~dB}$ up to $40 \mathrm{GHz}$ is reported, with a measured extracted $\mathrm{ON}$-state insertion loss of $0.52 \mathrm{~dB}$ at 35 $\mathrm{GHz}$, and less than $0.88 \mathrm{~dB}$ across the entire band from 30$40 \mathrm{GHz}$. The proposed switch illustrates the significant potential for photogenerated silicon plasma switching of high-performance bulk micromachined millimeter-wave waveguides.
\end{abstract}

Index Terms-Waveguide, switch, photogenerated solid-state plasma, millimeter-wave, silicon micromachining, deep reactive ion etching (DRIE).

\section{INTRODUCTION}

$\mathbf{M}$ ILLIMETER-WAVE switches are fundamental components of modern transceiver architectures, enabling a variety of functionality including signal routing, redundancy switching, band selection, and phase shifting. In particular, waveguide switches are typically chosen at millimeter-wave (mm-wave) frequencies and beyond due to their low insertion loss and high power handling [1]. A number of technologies for the switching element inside a waveguide have been developed, including electromechanical rotors and PIN diode switches [2]-[5], and RF MEMS and microfluidic-based switches [6]-[11]. However, most of these approaches use traditional metal-machined waveguides, thus are large, heavy, and difficult to integrate with planar microwave technology and on chip. While an integrated waveguide switch using RF MEMS was proposed in [7], self-actuation, reliability, and stiction issues limit its use for high power applications.

The optical control of microwave and mm-wave signals by photogeneration of solid-state plasma has shown promise as a high-performance switching and phase shifting technology in both planar, waveguide, and substrate integrated waveguide (SIW) technologies [12]-[14]. Not only is the bias network of the optical source isolated from the RF signal, the plasma element itself offers advantages including high power handling

T. R. Jones and D. W. Barlage are with the Department of Electrical and Computer Engineering, University of Alberta, AB, Canada, T6G1H9 e-mail: trjones@ualberta.ca.

A. Fisher and D. Peroulis are with Purdue University, IN, USA.

Manuscript received January 31, 2021. with low insertion loss [15], high linearity [16], and fast switching times [17]. Applications in the literature include switches, variable attenuators, and tunable phase shifters, [13], [18], [19].

While photogenerated solid-state plasma elements have been successfully integrated into rectangular waveguide and SIW technologies, the integration and measurement of a solidstate plasma switch inside a silicon micromachined waveguide channel at mm-wave frequencies has not yet been reported. Silicon bulk micromachining technology offers benefits including high precision with micrometer tolerances, low insertion loss, and high power handling up to sub-mm-wave bands and beyond, along with excellent integration with planar technologies [20], [21]. Furthermore, silicon micromachining technology enables the fabrication of both the solid-state plasma element and the waveguide channel with the same process for complete monolithic integration.

The main objective of this paper is to explore the use of photogenerated plasma as switching elements located inside silicon micromachined waveguides for operation as shuntbased microwave switches at mm-wave frequencies. To the best of the authors' knowledge, this is the first measured implementation of its kind, and highlights the significant potential of this technology for high performance mm-wave switching applications.

The paper is organized as follows. First, section II details the principle of operation of the photogenerated silicon plasma waveguide switch, with a simplified theoretical formulation to relate the applied optical power to the photogenerated conductivity of the silicon plasma. Next, in section III, the design procedure of a $\pi$-match waveguide switch using silicon plasma elements is detailed, with a description of the fabrication process flow using silicon bulk micromachining presented in section IV. The experimental results of the fabricated prototype are then presented in section $\mathrm{V}$, along with an analysis validating the theory derived in section II with measurement, and a discussion on the application of the waveguide switch as a variable attenuator. A comparison between state-of-theart millimeter-wave waveguide switches is presented in section VI, and final conclusions are then drawn.

\section{PRINCIPLE OF OPERATION}

\section{A. Fundamental Concept}

In Fig. 1(a), the fundamental concept behind the proposed photogenerated solid-state plasma waveguide switch is illus- 


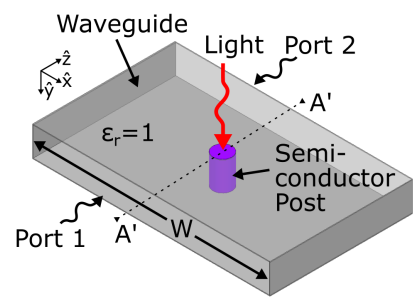

(a)

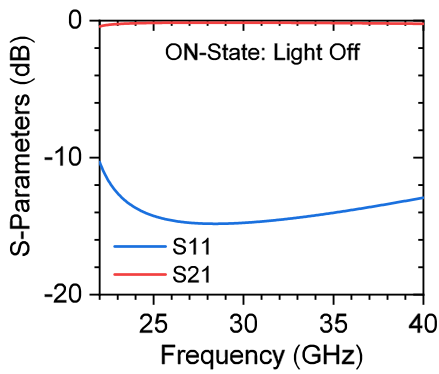

(c)

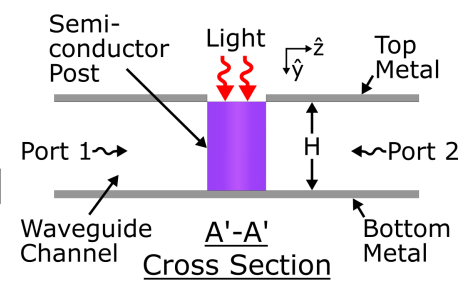

(b)

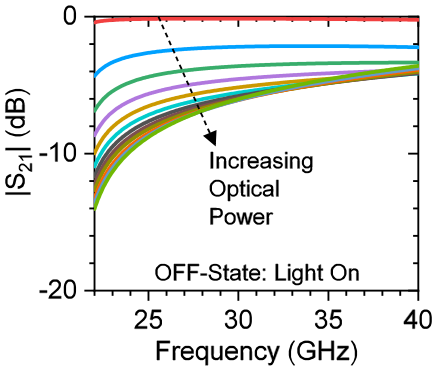

(d)
Fig. 1. (a) Conceptual waveguide switch 3D layout, and (b) cross sectional view A'-A' of the rectangular waveguide loaded with a semiconductor post for operation as a photogenerated solid-state plasma waveguide switch and variable attenuator, with HFSS simulations of the (c) ON-state, and (d) OFFstate with increasing optical power.

trated. A semiconductor post is placed within a rectangular waveguide channel in a shunt configuration, connected from the top of the waveguide to the bottom. The post is ideally located near the center due to the peak of the fundamental $\mathrm{TE}_{1,0}$ mode. As shown in Fig. 1(b), a small opening in the top of the waveguide allows photons from a light source with high enough energy to absorb into the semiconductor post, generating free carriers within. The semiconductor post is therefore changed from a capacitive to a conductive load, allowing for the transmission or reflection of $\mathrm{mm}$-waves within the waveguide channel, respectively.

The result of increasing the conductivity of the semiconductor post inside a waveguide channel as illustrated in Fig. 1(a) is simulated in HFSS. A waveguide with broad and narrow wall dimensions of $7.112 \mathrm{~mm}$ and $200 \mu \mathrm{m}$, respectfully, is chosen for operation within the Ka-band. The waveguide is loaded in the center with a semiconductor post of radius $200 \mu \mathrm{m}$ and dielectric constant $\epsilon_{r}=11.9$. The choice of reduced height waveguide is to allow the photo-excited plasma to extend the total height of the post $H$. The choice of permittivity matches that of silicon, a commonly available semiconductor, while the choice of post radius is due to the available optical fiber size for plasma excitation at the time of this work. More specific discussions on the choice of these values are presented below and in section III.

In Fig. 1(c), the ON-state scattering parameters with no optical excitation are shown, where the semiconductor is modeled as a dielectric element. Here the switch is in the transmission mode, with low insertion loss. Improving the ONstate return loss is discussed in section III.

In Fig. 1(d), the OFF-state of the switch is shown, whereby increasing the optical power incident on the top of the post increases the amount of isolation realized by the switch. Here, the post is modeled in HFSS as a lossy dielectric with a specified conductivity, and a maximum isolation is reached at a conductivity between $350-400 \mathrm{~S} / \mathrm{m}$. Further increasing the conductivity improves the total system loss, i.e., more waves are reflected instead of dissipated in the post. This effect, and methods to improve the isolation, are discussed further in section $\mathrm{V}$ and section III, respectively.

From this simple proof of principle, clearly the primary goal is to maximize the amount of on/off ratio versus optical energy on the post. Therefore, a material with good semi-insulating properties to drive the ON-state, and optical excitation properties to drive the OFF-state, is desired. Two such materials which have been explored in the literature for optical switches are high resistivity intrinsic silicon and germanium [22]. Both offer long carrier lifetimes due to their indirect bandgaps, enabling higher conductivity at lower optical energies for the OFF-state. However, germanium generally has poorer semiinsulating properties than silicon for the $\mathrm{ON}$-state insertion loss [23], as well as less availability for purchasing high quality substrates. Therefore, due to long carrier lifetimes, reasonable resistivities, greater availability of high insulating substrates at low cost, along with well developed bulk micromachining processes, high resistivity $(>10 \mathrm{k} \Omega \mathrm{cm})$ intrinsic silicon was determined to be the most reasonable choice for the present work, and will be referred to going forward.

\section{B. Photoconductivity Model}

To quantify the relationship between the optical power incident on the top surface of the silicon post and the photogenerated conductivity and permittivity within the bulk, a theoretical analysis is now developed. This will allow for a quick and accurate calculation for the silicon plasma design parameters to be used in a 3D full-wave simulation tool such as HFSS. Validation of the analysis is presented in section V, where an excellent match between the theory developed here and the experimental results of the waveguide switch is shown.

When light is incident on the surface of silicon with photon energy $h \nu$ greater than the bandgap $\left(E_{g}=1.12 \mathrm{eV}\right)$, electronhole pairs are generated within the bulk, and the silicon enters a non-equilibrium state. The carrier concentrations within the silicon are then expressed as

$$
\begin{aligned}
& n=n_{0}+n^{\prime} \\
& p=p_{0}+p^{\prime}
\end{aligned}
$$

where $n, p$ are the total, $n_{0}, p_{0}$ the intrinsic, and $n^{\prime}, p^{\prime}$ the photogenerated excess concentrations of electrons and holes, respectively. For high-carrier injection, the typical case for the present application, $n^{\prime} \gg n_{0}$ and $p^{\prime} \gg p_{0}$. Furthermore, the number of photogenerated electrons and holes are equal, such that $n^{\prime}=p^{\prime}$.

In order to simplify the analysis, the silicon is treated as a bulk volume in which a steady flux of photons $\phi_{\text {opt }}$ at optical wavelength $\lambda_{\text {opt }}$ incident on the top of the post generates a uniform carrier concentration throughout. This is a reasonable assumption if the depth of the post $H$ is larger than the absorption depth $1 / \alpha$ [24], where the absorption coefficient $\alpha$ 
can be calculated from $\alpha=4 \pi \kappa_{s} / \lambda_{o p t}$, with $\kappa_{s}$ the imaginary part of the complex refractive index $\left(n_{r}=n_{s}-j \kappa s\right)$ at $\lambda_{o p t}$ [24]. Given the optical source shown in Fig. 1(a) with a wavelength $\lambda_{o p t}=915 \mathrm{~nm}$ and $\kappa_{s}=1.894 \times 10^{-3}$ [25], the calculated absorption coefficient $\alpha=250 \mathrm{~cm}^{-1}$. Thus, at a depth of $H=200 \mu \mathrm{m}, 99.4 \%$ of the photon flux is absorbed by the silicon. It is then reasonable to expect the carriers to diffuse throughout the enclosed volume of the post within a time scale based on the thermal velocity $v_{t h}=\sqrt{3 k T / m^{*}}$, where $k$ is the Boltzmann constant, $T$ the ambient temperature, and $m^{*}$ the effective mass, creating a uniform carrier concentration throughout.

Considering the 1D case where the top of the silicon post shown in Fig. 1 is uniformly illuminated by light, with variation of the photon flux density only in the $\hat{y}$ direction, the steady-state generation $\left(G_{e}\right)$ and recombination $\left(R_{e}\right)$ rates of the electron-hole pairs can then expressed by the following relationships [24]

$$
\begin{gathered}
G_{e}=\frac{\eta \phi_{o p t}(y)}{H} \\
R_{e}=\frac{n^{\prime}}{\tau\left(n^{\prime}\right)} \\
G_{e}=R_{e} .
\end{gathered}
$$

Here, $\tau\left(n^{\prime}\right)$ is the carrier lifetime as a function of carrier concentration, and $\eta$ the quantum efficiency (number of electronhole pairs generated per photon).

Considering an optimal efficiency $(\eta=1)$, the photon flux density can be expressed as [24]

$$
\phi_{\text {opt }}(y)=P_{\text {opt }}(y) / h \nu
$$

where the power intensity $P_{o p t}$ is determined from [24]

$$
P_{\text {opt }}(y)=P_{0}(1-R) e^{-\alpha y}
$$

with $P_{0}=P / A$ the incident light intensity on the surface of the silicon, $P$ the incident optical power, $A=\pi d^{2}$ the surface area of the top of the post with diameter $d$, and $R=\left\{\left(1-n_{s}\right)^{2}+\kappa_{s}^{2}\right\} /\left\{\left(1+n_{s}\right)^{2}+\kappa_{s}^{2}\right\}$ the reflection coefficient at the silicon interface with normal incidence. Therefore, based on the earlier assumption of a uniform carrier concentration throughout the silicon post, the average power intensity absorbed into the post can be calculated as

$$
\overline{P_{o p t}}=\frac{1}{H} \int_{0}^{H} P_{o p t}(y) d y=\frac{P_{0}}{\alpha H}(1-R)\left(1-e^{-\alpha H}\right)
$$

and the uniform carrier concentration throughout the post can be determined from

$$
n^{\prime}=\frac{P_{0} / h \nu}{\alpha H^{2}} \tau\left(n^{\prime}\right)(1-R)\left(1-e^{-\alpha H}\right)
$$

With respect to (9), the carrier concentration is proportional to the incident optical power intensity, the carrier lifetime, and one minus the reflection coefficient. To improve the number of carriers generated, the reflected power can be decreased by depositing anti-reflection coatings (ARC) on the surface of the silicon. Furthermore, the optical power intensity can be increased. However, it is well known that the carrier lifetime begins to rapidly decrease at higher carrier concentrations $\left(\sim 10^{16-17} \mathrm{~cm}^{-3}[23]\right)$ primarily due to Auger recombination [24], [26]. To model this effect, the following equation is used to represent the carrier lifetime as a function of carrier concentration [27]

$$
\tau\left(n^{\prime}\right)=\frac{\tau_{0}}{1+\left(n^{\prime} / N_{0}^{r e f}\right)^{3 / 2}}
$$

where $\tau_{0}$ and $N_{0}^{r e f}$ are fitting coefficients. Thus, the most important parameter to consider when designing for the maximum on/off ratio of the post is the carrier lifetime, which is largely related to the quality, or maximum resistivity, of the intrinsic silicon material. Typical values for $\tau_{0}$ and $N_{0}^{r e f}$ in the literature are $0.1-1000 \mu \mathrm{s}$ and $10^{15}-10^{17} \mathrm{~cm}^{-3}$, respectively [26], [27].

With the carrier concentration now known, the DC photoconductivity of the silicon post can be determined from [24]

$$
\sigma_{D C}=q\left(\mu_{n}+\mu_{p}\right) n^{\prime}
$$

where $\mu_{n}, \mu_{p}$ are the electron and hole drift mobilities, respectively, and $q$ is the unit charge of an electron. Thus, the contribution to the total DC photoconductivity from electrons and holes adds in parallel. Furthermore, the drift mobilities are themselves functions of carrier concentration, and can be calculated for a given $n^{\prime}$ using the following [28]

$$
\begin{aligned}
& \mu_{n}=\frac{1318}{1+\left(n^{\prime} / 1 \times 10^{17}\right)^{0.85}}+92 \\
& \mu_{p}=\frac{420}{1+\left(n^{\prime} / 1.6 \times 10^{17}\right)^{0.7}}+50
\end{aligned}
$$

As free carriers are generated in the silicon bulk, along with the change in conductivity, a change in the dielectric constant occurs. This effect can be modelled using the Drude response in terms of the intrinsic bulk complex conductivity of the silicon plasma given by [29], [30]

$$
\sigma(\omega)=\frac{\sigma_{D C}}{1-j \omega / \gamma_{n, p}}=\sigma^{\prime}(\omega)-j \sigma^{\prime \prime}(\omega)
$$

where $\gamma_{n, p}=\left(m_{n, p}^{*} \mu_{n, p} / q\right)^{-1}$ is the average collision rate, and $m_{n, p}^{*}$ the effective mass, of an electron or hole, respectively. Thus, the real and imaginary components of $\sigma(\omega)$ can be expressed as the following

$$
\sigma^{\prime}(\omega)=\frac{\sigma_{D C}}{1+\omega^{2} / \gamma_{n, p}^{2}}, \sigma^{\prime \prime}(\omega)=\frac{\sigma_{D C} *\left(\omega \gamma_{n, p}^{-1}\right)}{1+\omega^{2} / \gamma_{n, p}^{2}}
$$

At operating frequencies below $100 \mathrm{GHz}, \gamma_{n, p} \gg \omega$, and the real component $\sigma^{\prime}(\omega)$ is approximately equal to the DC photoconductivity $\sigma_{D C}$ calculated in (11). Using the well known relationship between intrinsic bulk permittivity $\epsilon(\omega)$ and intrinsic complex conductivity $\sigma(\omega)=j \omega \epsilon(\omega)$, the effective dielectric constant $\epsilon_{r}(\omega)$ of the silicon plasma can be determined from the following [30]

$$
\epsilon_{r}(\omega)=\epsilon_{\infty}-\sigma^{\prime \prime}(\omega) / \omega \epsilon_{0}
$$

where $\epsilon_{\infty}$ is the background dielectric constant for silicon $(11.9,[24])$, and $\epsilon_{0}$ is the permittivity of free-space. Thus, to simulate the photo-excited silicon material in HFSS, the effective dielectric constant $\epsilon_{r}$ and DC photoconductivity $\sigma_{D C}$ are entered into their respective material fields for relative permittivity and bulk conductivity [30]. 


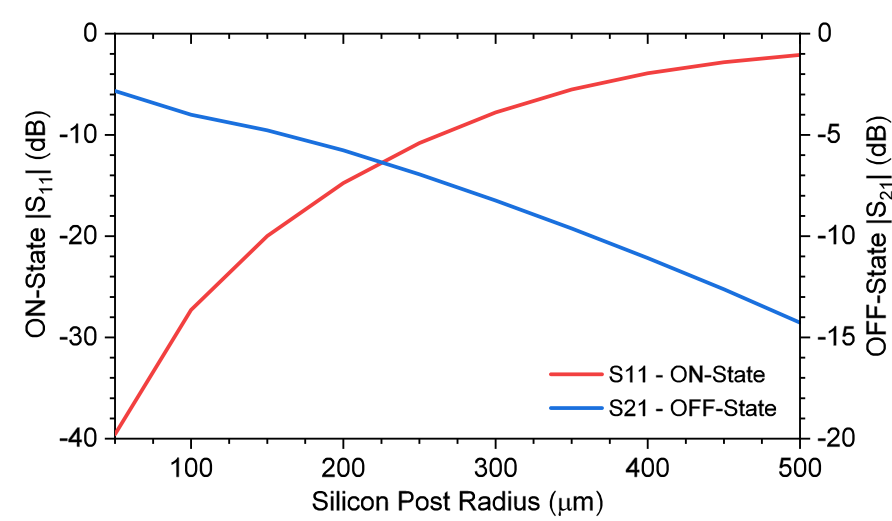

Fig. 2. HFSS simulation results of the ON-state return loss and OFF-state isolation at $30 \mathrm{GHz}$ versus silicon post radius for the waveguide switch model shown in Fig 1.

\section{DESIGN}

This section describes the design of a silicon plasma waveguide switch. Due to the high permittivity of the silicon post located within the waveguide channel shown in Fig. 1(a), the ON-state of the waveguide switch suffers large reflection losses caused by the large shunt capacitive loading of the post. Fig. 2 shows the effect on the ON-state return loss and OFFstate isolation versus the radius of the post for the waveguide switch in Fig. 1(a). By increasing the radius, more isolation is achieved at the expense of impedance matching, and vice versa. Increasing the number of posts in the transverse plane would have a similar effect. It is clear that a trade-off between $\mathrm{ON}$-state return loss and OFF-state isolation must be made, thus maximizing the on/off ratio becomes difficult with such a switch configuration.

For a more optimal way to increase the on/off ratio for switches with high ON-state capacitance, a $\pi$-match switch topology can be chosen [31]. A high impedance section is inserted between two shunt switch elements to improve ONstate matching. The required characteristic impedance value $Z_{h}$ of the high impedance section can be calculated from the following equation [31]

$$
\tan (\beta l)=\frac{2 \omega_{0} C_{u} Z_{h} Z_{0}^{2}}{Z_{h}^{2}-Z_{0}^{2}+\left(\omega_{0} C_{u} Z_{h} Z_{0}\right)^{2}}
$$

where $\beta$ is the propagation constant and $l$ the propagation length of the high impedance waveguide section, $Z_{0}$ the characteristic impedance of the main waveguide section, and $\omega_{0}$ the angular frequency at band center. To relate the waveguide height $H_{h}$ and width $W$ of the high impedance section to its characteristic impedance, the following power-current definition for an air-filled waveguides is used [32]

$$
Z_{h}=\frac{465 H_{h} \lambda_{g}}{W \lambda}
$$

where $\lambda_{g}$ is the guided wavelength and $\lambda$ the free-space wavelength at the band center frequency. Thus, the high impedance section can be formed by simply increasing the height of the waveguide section between the two shunt switches.

In Fig. 3, the final design layout including dimensions for microfabrication of the waveguide switch is shown. The

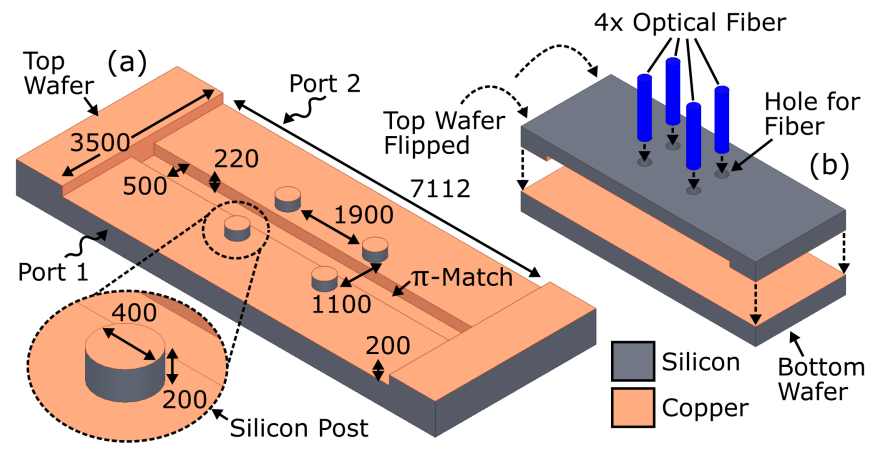

Fig. 3. П-design layout of the photogenerated silicon plasma waveguide switch showing (a) the top wafer underside with silicon posts and $\pi$-match network, and (b) top wafer flipped onto bottom wafer for bonding (units in $\mu \mathrm{m})$.

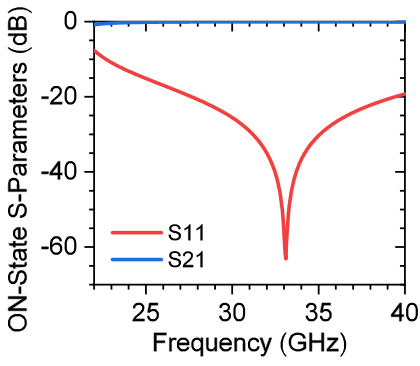

(a)

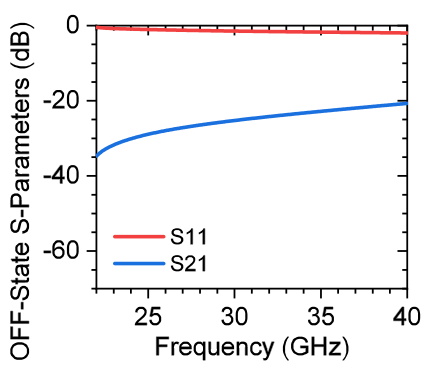

(b)
Fig. 4. HFSS simulation results of the (a) ON-state and (b) OFF-state of the silicon plasma $\pi$-match waveguide switch shown in Fig. 3 .

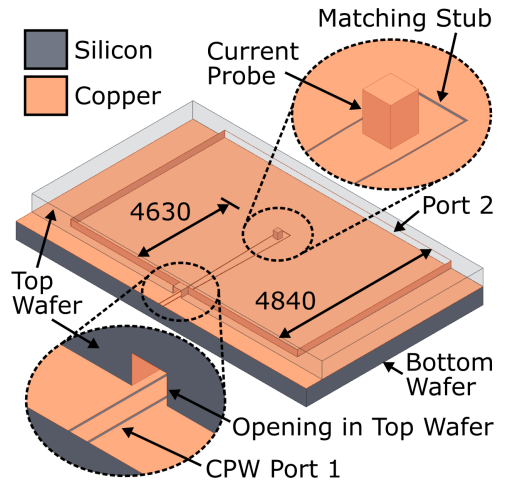

(a)

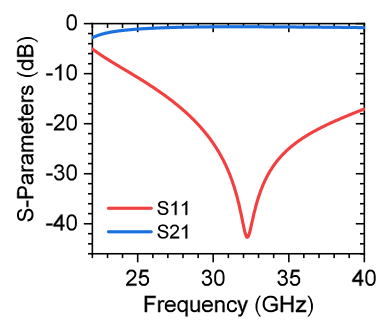

(b)
Fig. 5. (a) 3D layout of the CPW-to-waveguide feed network for characterization of the waveguide switch using high frequency probes (units in $\mu \mathrm{m}$ ), and (b) HFSS simulation results of the transition.

prototype was chosen to operate within the Ka-band, with a total waveguide width of $7.112 \mathrm{~mm}$ (WR28). The design was limited to four silicon posts due to the availability of a $1 \times 4$ optical fiber splitter, with four individual fibers supplying the optical excitation. Therefore, to achieve greater than 20 dB OFF-state isolation, a 2-by-2 array of posts with 400 $\mu \mathrm{m}$ diameters was placed within the waveguide channel. A high impedance $\pi$-match section was designed in between the posts to improve ON-state impedance matching, while also decreasing the electrical length of the overall switch. The top wafer was then flipped and bonded to the bottom wafer, as 


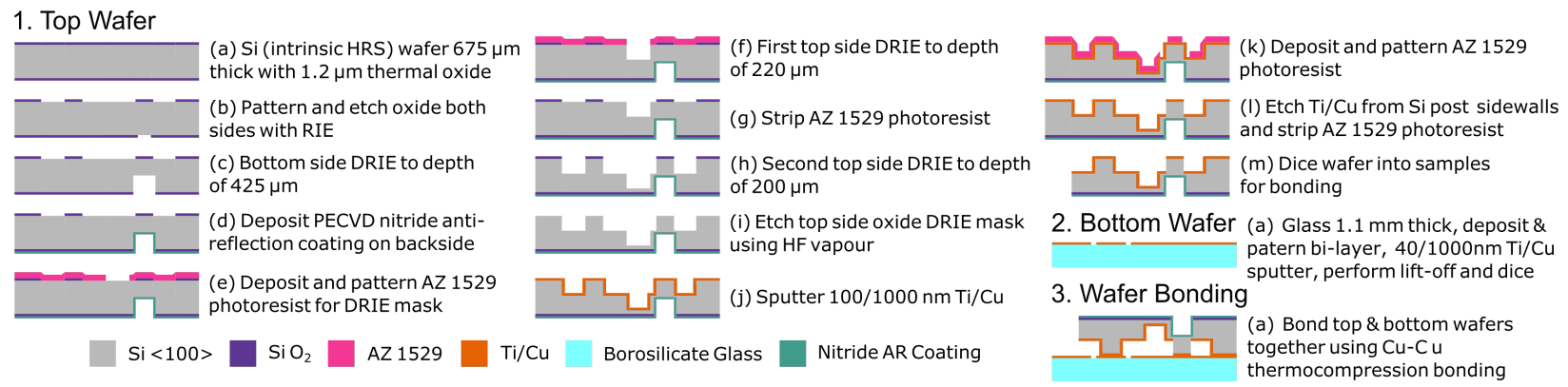

Fig. 6. Fabrication process flow for the waveguide switch.

shown in Fig. 3(b). Four optical fibers inserted into holes etched in the backside of the top wafer directly above the silicon posts provided optical excitation of the silicon plasma.

As discussed in section II, a longer carrier lifetime results in a silicon post with a higher conductivity. Thus, a high resistivity $(>10 \mathrm{k} \Omega \mathrm{cm})$ intrinsic silicon wafer was chosen with a thickness of $675 \mu \mathrm{m}$ to allow for the height of the high impedance section, and the backside etching of slots to align the fibers. The height of the waveguide channel was chosen to be $200 \mu \mathrm{m}$ to allow for the silicon posts to have high enough conductivity for good isolation in the OFF-state, while also being large enough to limit conductor losses due to the reduced height waveguide in the ON-state.

In Fig. 4, the HFSS simulated results are plotted for the ON-state and OFF-state of the final prototype shown in Fig. 3. The ON-state return loss is above $20 \mathrm{~dB}$ from $27.6 \mathrm{GHz}$ to $39.5 \mathrm{GHz}$, with a reflection zero at $33.1 \mathrm{GHz}$ due to the $\pi$-match network. The OFF-state was simulated with a silicon post conductivity of $1000 \mathrm{~S} / \mathrm{m}$ and dielectric constant $\epsilon_{r}=-2$, according to (16). The isolation is greater than $20 \mathrm{~dB}$ across the entire band up to $40 \mathrm{GHz}$.

In order to characterize the waveguide switch using high frequency RF probes, a coplanar waveguide (CPW)-to-waveguide feed network was designed, based on a current probe topology [33]. Fig. 5(a) shows the 3D layout of the CPW-to-waveguide transition. A CPW trace with $120 \mu \mathrm{m}$ signal and $15 \mu \mathrm{m}$ gap was designed for probing, which then enters an opening in the waveguide short-circuit wall. The CPW then tapers to a signal line width of $272 \mu \mathrm{m}$ and $9 \mu \mathrm{m}$ gap before it reaches a current probe, which extends from the bottom CPW trace to the top of the waveguide channel. The distance from the current probe to the waveguide back wall short-circuit is approximately $\lambda_{g} / 4$ at the band center frequency. A small open-circuit stub on the other side of the current probe can be used to help tune the impedance match. The HFSS simulation results of the transition are plotted in Fig. 5(b), where good matching is achieved from 30 to $40 \mathrm{GHz}$. At $35 \mathrm{GHz}$, the simulated insertion loss of the transition is $0.67 \mathrm{~dB}$.

\section{FABRICATION}

The fabrication process of the waveguide switch is shown in Fig. 6. Silicon micromachining using deep reactive ion etching (DRIE) was chosen for its capability to achieve both small and large features with high precision [21]. The process starts with a high resistivity intrinsic silicon wafer $675 \mu \mathrm{m}$ thick with a
$1.2 \mu \mathrm{m}$ thermal oxide grown on both topside and backside faces (1a). The oxide was patterned on both sides for the topside and backside DRIE masks (1b), where the topside etch was used to pattern the waveguide, silicon post, and waveguide feed network, and the backside etch was used to pattern cavities to insert and align the optical fibers directly above the silicon posts. The cavities for the fiber were then etched into the backside using DRIE (1c), and a PECVD nitride was deposited as an anti-reflection coating (1d). Next, to achieve two different topside etch depths for the main waveguide channel and high impedance slot of the $\pi$-match network, AZ 1529 photoresist was first patterned above the topside oxide (1e), and the high impedance $\pi$-match slot was etched (1f). The resist was stripped (1g), and the main waveguide channel was etched (1h). The topside oxide mask was stripped to improve the electrical connection of the post (1i). Deposition of a sputtered 100/1000 nm Ti/Cu layer metalizes the topside waveguide channel $(1 \mathrm{j})$, and the metal from the sidewalls of the silicon posts were removed by patterning AZ 1529 (1k) and wet chemical etching (11). The individual samples were then diced $(1 \mathrm{~m})$. For this prototype, a borosilicate glass wafer $1.1 \mathrm{~mm}$ thick was chosen for the bottom carrier wafer and CPW feed network. A 40/1000 nm Ti/Cu layer was patterned using a MicroChem LOR 5B and AZ 1512 bi-layer lift-off process, and the individual samples were diced (2a). Finally, the top and bottom wafers were bonded together using $\mathrm{Cu}-\mathrm{Cu}$ thermocompression bonding (3a).

\section{EXPERIMENTAL RESUlTS}

\section{A. Experimental Setup}

Fig. 7 shows the experimental setup for characterization of the $\pi$-match waveguide switch designed in section III. The device was measured using an Agilent E8361C PNA with an N5260A millimeter head controller, N5260-60003/60004 test head modules, and Cascade Microtech Summit 11000 probe station with Z-probes. The optical setup included a $915 \mathrm{~nm}, 7$ watt Sheaumann Laser Inc. laser diode [34] in a TO220 package pig-tailed with a $100 \mu \mathrm{m}$ fiber and attached to a general purpose heatsink with thermal paste. The optical source at $\lambda_{o p t}=915 \mathrm{~nm}\left(h \nu_{o p t}=1.36 \mathrm{eV}\right)$ was chosen to be within the range of high quantum efficiency for silicon [35]. A Wavelength Electronics TCS610 $10 \mathrm{kOhm}$ thermistor was inserted underneath the laser diode to monitor the operating temperature. The laser diode was driven by a Wavelength 


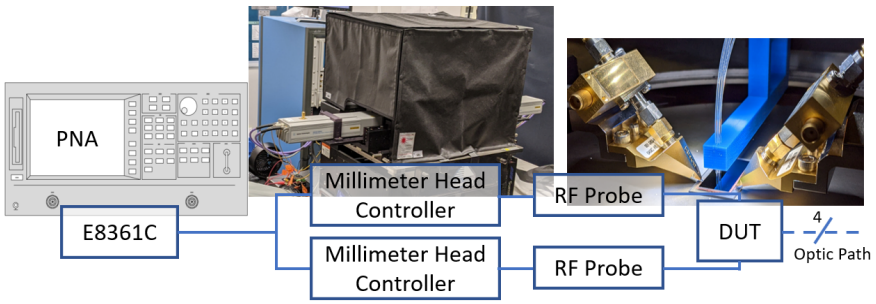

(a)

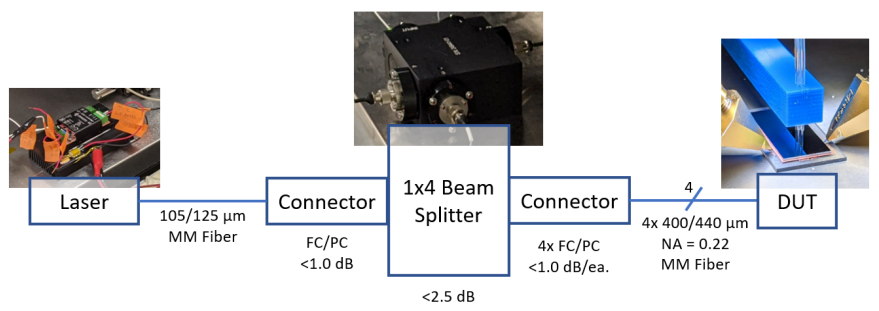

(b)

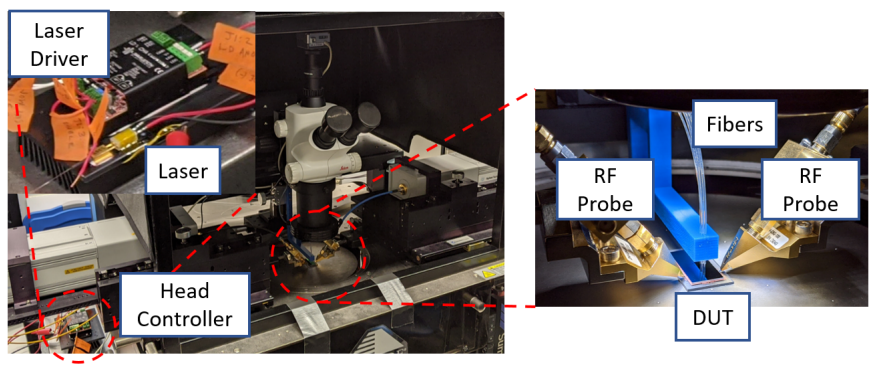

(c)

Fig. 7. Experimental setup with (a) device characterization using a high frequency probe station, (b) optical fiber setup including beam splitter and connector losses, and (c) close up of the laser diode driver and device under test (DUT).

Electronics LD5CHA 5 A constant current laser diode driver [36] attached to a general purpose heatsink with thermal paste. The laser diode driver was powered by a Wavelength Electronics PWRPAK-5V $+5 \mathrm{~V}$ switching power supply with less than $1 \%$ peak-to-peak input ripple and noise. In order to supply four separate fibers to the $\pi$-match switch, the $100 \mu \mathrm{m}$ fiber was split into four $400 \mu \mathrm{m}$ fibers using a non polarizing beam splitter with $25 \%$ power ratio from OZ Optics [37]. The estimated optical loss as given by the manufacturer for each component is shown in Fig. 7(b). The total loss at the output of each $400 \mu \mathrm{m}$ fiber versus input optical energy from the laser diode, including the $25 \%$ power-ratio splitting, is estimated to be less than $10.5 \mathrm{~dB}$. The choice of optical setup was mostly due to availability and experimental convenience. A more integrated setup could be employed in the future with less optical loss, using microLEDs or vertical-cavity surfaceemitting lasers (VCSEL).

\section{B. Measured Results}

In Fig. 8, pictures of the fabricated waveguide switch prototype are presented. The top and bottom wafer pieces before bonding are shown in Fig. 8(a), highlighting the switch region, the CPW-to-waveguide transition region for characterization, and the back-to-back waveguide without silicon posts or $\pi$ -

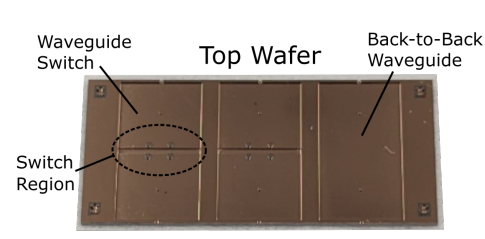

Bottom Wafer

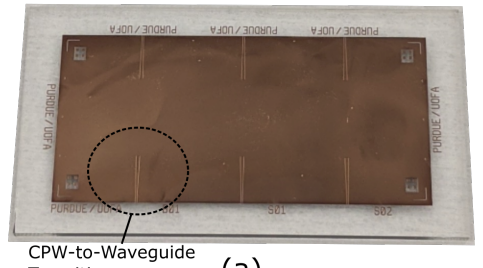

Transition (a)

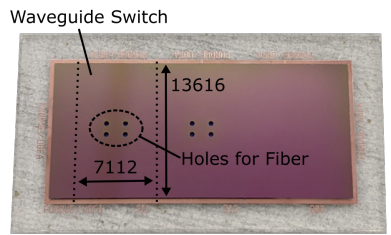

(b)

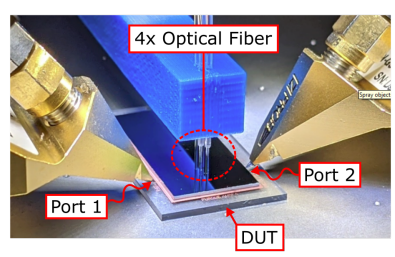

(c)
Fig. 8. Picture of the (a) top and bottom wafers highlighting the switch region, CPW-to-waveguide transition, and the back-to-back waveguide without silicon posts or $\pi$-match network, (b) the device after bonding (units in $\mu \mathrm{m}$, and (c) the measurement of the device using two FormFactor $|\mathrm{Z}|$ Probes, with four optical fibers inserted into the four holes etched in the backside of the top wafer located above each silicon post.

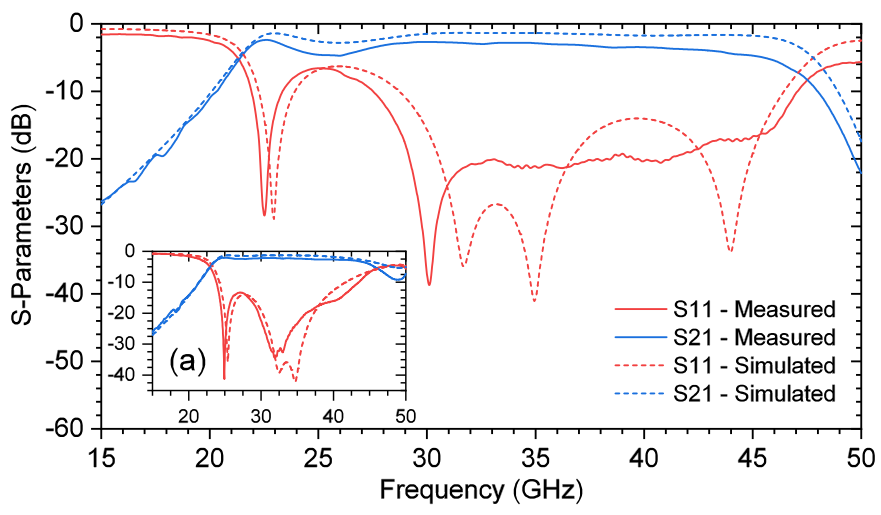

Fig. 9. Measured and simulated $\mathrm{ON}$-state scattering parameters for the waveguide switch, with inset (a) the back-to-back structure (with no silicon posts).

match network for switch loss extraction. Fig. 8(b) shows the bonded waveguide switch, highlighting the hole locations for the optical fibers, and final dimensions of the waveguide switch including transitions. In Fig. 8(c), a picture of the probe measurement is shown, with the four optical fibers inserted into the holes located directly above the silicon posts.

In Fig. 9, the measured ON-state scattering parameters of the $\pi$-match waveguide switch are compared with the simulation results from HFSS, including CPW-to-waveguide transitions. The measured switch has return loss greater than $20 \mathrm{~dB}$ from $29.2-41.6 \mathrm{GHz}$, and return loss greater than 10 $\mathrm{dB}$ from $27.4-47.2 \mathrm{GHz}$. At $35 \mathrm{GHz}$, the measured insertion loss is $2.86 \mathrm{~dB}$, with a minimum and maximum insertion loss of $2.81 \mathrm{~dB}$ and $3.71 \mathrm{~dB}$, respectively, within the $20 \mathrm{~dB}$ return loss bandwidth.

To extract the loss of the switch element by itself, the back-to-back waveguide structure without the silicon posts and $\pi$-match network shown in Fig. 8 was measured, with its scattering parameters shown in Fig. 9(a). The extracted ONstate insertion loss of the waveguide switch element is then 
found by subtracting the insertion loss of the back-to-back structure from the insertion loss of the waveguide switch. With a measured back-to-back insertion loss of $2.34 \mathrm{~dB}$ at $35 \mathrm{GHz}$, the measured extracted insertion loss of the switch is $0.52 \mathrm{~dB}$, while the net impact of the switch element across the entire band from $30 \mathrm{GHz}$ to $40 \mathrm{GHz}$ is below $0.88 \mathrm{~dB}$.

The discrepancy between measured and simulated return loss is likely due to the fabrication tolerances of the silicon DRIE processes shown in Fig. 6. First, the fabricated sidewalls had a measured negative slope of $2.8^{\circ}$. Second, the etch depths for both the waveguide channel and high impedance slot had measured tolerances of approximately $\pm 5 \%$ across the wafer. With target etch depths of $200 \mu \mathrm{m}$ and $220 \mu \mathrm{m}$, respectively, this led to a variability of $\pm 10 \mu \mathrm{m}$ and $\pm 11 \mu \mathrm{m}$ for the final height of the two regions, respectively.

The discrepancy between the measured and simulated insertion loss is likely due to the overall thickness and skin depth of the sputtered copper across the wafer, especially considering the thickness of the sidewall coverage will generally be less than the horizontal surfaces. The conductivity of the sputtered copper was measured using a four-point probe, with a value of $\sigma=2.3 \times 10^{7} \mathrm{~S} / \mathrm{m}$. Thus, at $35 \mathrm{GHz}$, the skin depth is approximately $561 \mathrm{~nm}$. Increasing the sputtered copper thickness from $1 \mu \mathrm{m}$ to greater than $3 \times$ the calculated skin depth would help decrease insertion loss.

Furthermore, the period of time between sputtering and bonding allows the growth of a copper oxide. The effect of this oxide was determined to be most significant at the current probe of the CPW-to-waveguide transition. To mitigate this effect, a quick 10 second dip in diluted hydrochloric acid solution right before the bonding step in Fig. 6 was carried out during fabrication to remove the oxide layer [38]. However, a certain amount of oxide will always be present due to a non-negligible amount of time the sample spends in atmosphere between the acid dip and bonding step, while the acid dip also slighlty increases surface roughness. This effect can be mitigated for industrial applications with proper levels of process control, i.e., keeping the copper in an inert environment at all times.

In Fig. 10, the measured OFF-state (a) isolation $\left(\left|S_{21}\right|\right)$ and (b) return loss $\left(\left|S_{11}\right|\right)$ of the waveguide switch are shown with increasing optical power at the output of each fiber. At $35 \mathrm{GHz}$, an isolation of $20 \mathrm{~dB}$ and return loss of $9 \mathrm{~dB}$ is achieved at an optical power of $50.1 \mathrm{~mW}$ per fiber, while an isolation of $25 \mathrm{~dB}$ and return loss of $6 \mathrm{~dB}$ is achieved at an optical power of $166.7 \mathrm{~mW}$ per fiber. This is significant isolation for this frequency range, and clearly demonstrates the potential of this technology for mm-wave switch applications.

It is noted in Fig. 10 that with increasing optical power above $166.7 \mathrm{~mW}$ up to $332.1 \mathrm{~mW}$, the isolation improves by only $0.51 \mathrm{~dB}$. Thus, a saturation point is reached, where any further increase in optical power no longer returns a significant change in isolation. However, the post continues to become more conductive, reducing the total loss in the system, which will be discussed in the next section. To further improve OFFstate isolation, additional posts can be added, keeping in mind the need to compensate for an increase in ON-state capacitive loading with the $\pi$-match region.

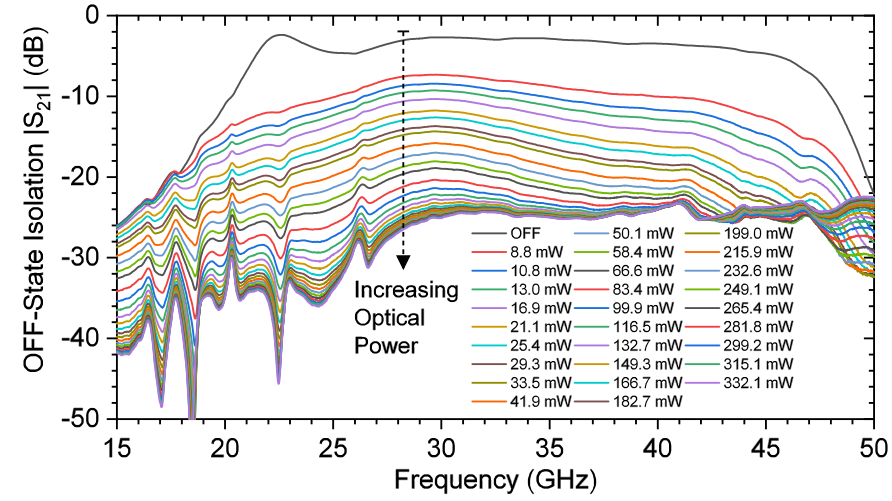

(a)

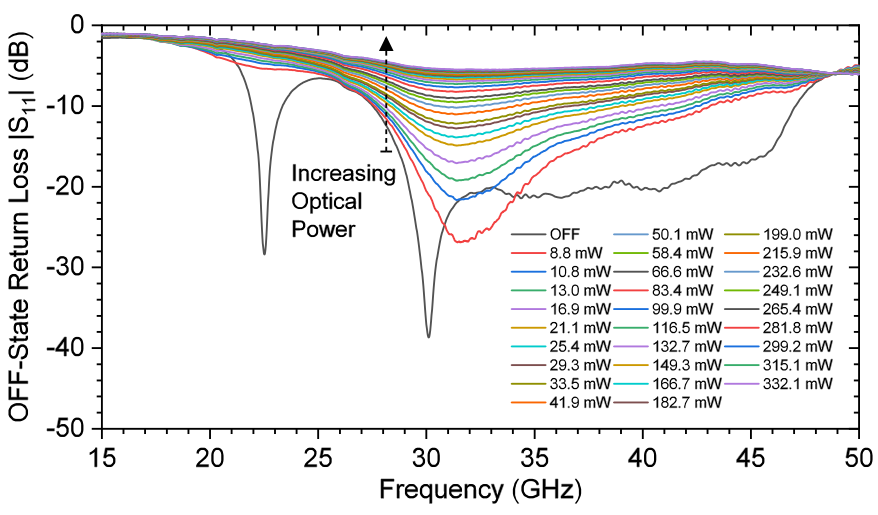

(b)

Fig. 10. Measured OFF-state (a) isolation $\left(\left|S_{21}\right|\right)$, and (b) return loss $\left(\left|S_{11}\right|\right)$ of the waveguide switch with increasing optical power. The value shown is the power output of each individual fiber for the two-by-two post array as shown in Fig. 3.

\section{Theoretical Validation}

The theory derived in section II is now used to correlate the measured waveguide switch performance versus applied optical power to the simulated performance versus the conductivity of the silicon posts. As described in section II, the photo-excited silicon elements simulated in HFSS are treated as a material with real conductivity and permittivity, where the permittivity for a given conductivity is determined from (16) [30]. To isolate the effect of losses due to the silicon plasma from losses associated with the copper conductor, the $\mathrm{ON}$-state loss is subtracted from the OFF-state loss at each applied optical power level (or each simulated conductivity), with the respective loss calculated using the equation [31]

$$
\text { Loss }=1-\left|S_{11}\right|^{2}-\left|S_{21}\right|^{2} .
$$

In Fig. 11, the extracted losses of the measured and simulated waveguide switch are plotted, with inset (a) showing the correlation between the photogenerated conductivity versus applied optical power. By adjusting the fitting coefficients of the carrier lifetime in (10), a good match between simulation and measurement is achieved with values $\tau_{0}=6.2 \mu \mathrm{s}$ and $N_{0}^{r e f}=1.1 \times 10^{16} \mathrm{~cm}^{-3}$, which are within the respective ranges for silicon. As the optical power begins to increase, a peak in the extracted loss occurs at approximately $69 \mathrm{~S} / \mathrm{m}$. Here, the silicon post is in its most lossy state, behaving 


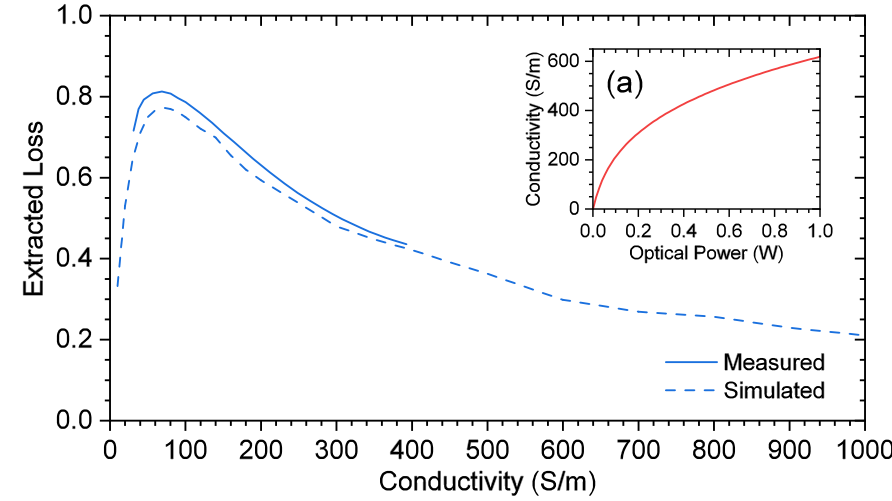

Fig. 11. Measured and simulated extracted loss at $35 \mathrm{GHz}$, with inset (a) the correlation between photogenerated conductivity versus applied optical power.

closer to an absorptive switch. Further increasing the optical power decreases the loss, with more power reflected due to the increase in conductivity of the silicon plasma. Furthermore, it is interesting to note that the above technique can be used as a reasonably effective method to characterize the carrier lifetime of silicon at microwave and millimeter-wave frequencies.

\section{Application as a Variable Attenuator}

As is shown in Fig. 10, by adjusting the amount of optical power, a variable attenuator can be achieved with the proposed waveguide switch. Therefore, it is important to know how the transmission phase delay and group delay change versus the level of attenuation.

Fig. 12(a) plots the phase of $S_{21}$ versus frequency for a few different optical power levels along with the measured unloaded back-to-back waveguide. With greater optical power, the amount of phase lag at $13.0 \mathrm{~mW}$ increases compared to the ON-state (laser OFF). However, at $50.1 \mathrm{~mW}$ and above, the amount of phase lag decreases compared to the ON-state. In addition, the phase lag of the switch is lower than the phase lag of the back-to-back waveguide above approximately 83.4 $\mathrm{mW}$. There is also a slight increase in the slope from $30 \mathrm{GHz}$ to $40 \mathrm{GHz}$ at all power levels other than the ON-state, indicating a dispersion effect when the silicon post is in its excited state.

In Fig. 12(b), the measured and simulated $S_{21}$ group delay versus frequency are shown for the ON-state (laser OFF), the OFF-state at the maximum applied power level, and the OFFstate at an intermediate power level showing the minimum achieved group delay, along with the unloaded back-to-back waveguide. The group delay $\tau_{g}$ is calculated using the equation [39]

$$
\tau_{g}=d \phi / d \omega
$$

where $\phi$ is the phase of $S_{21}$, and $\omega$ the angular frequency. The $S_{21}$ phase data for each measured trace was pre-fit with a $5^{\text {th }}$ order polynomial to reduce the systematic calibration error. The fitted data was then used to calculate the group delay.

A clear decrease in group delay is observed, indicating that with increased optical power, the group velocity of the waveguide switch becomes greater than the group velocity of the unloaded back-to-back waveguide. As the switch transitions

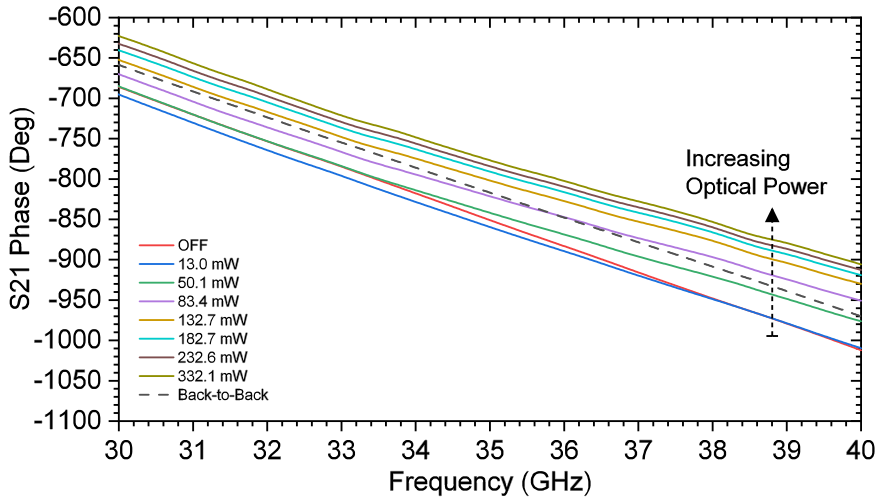

(a)

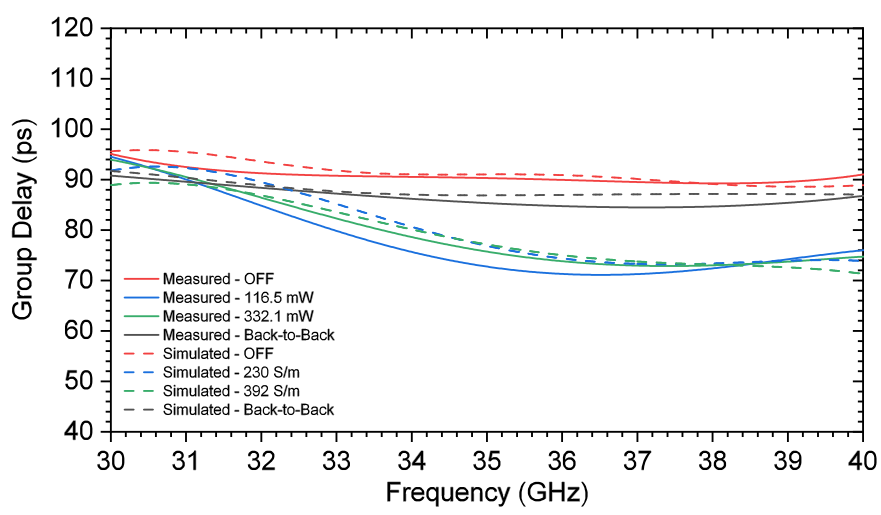

(b)

Fig. 12. (a) Measured $S_{21}$ phase, and (b) measured and simulated $S_{21}$ group delay versus frequency of the waveguide switch at different optical powers. The phase and group delay of the back-to-back waveguide (without silicon posts and $\pi$-match network) are also shown for comparison.

between the ON-state to the OFF-state, within the transition, an evanescent mode effect inside a waveguide channel is know to give faster group velocity than the unloaded case [40].

It is also worth mentioning the significant jump in the level of attenuation from the ON-state to the lowest power OFFstate in Fig. 10. This is likely due to the sensitivity of the laser diode driver used in experiment. Having more sensitive control of the drive current for the laser diode would allow lower optical power levels, and thus attentions, to be reached.

\section{DISCUSSION}

Table I compares the performance of the presented photogenerated silicon plasma waveguide switch to the state-ofthe-art in millimeter-wave waveguide switches, grouped by electrical (top) and mechanical (bottom) based technologies. While electromechanical switches achieve very good insertion loss and isolation, they suffer slow switching speeds in the millisecond range and are generally large in size [3]. The RF MEMS switches achieve the lowest insertion losses with good isolation, yet their fabrication is more complex and their switching speed is in the high 10s of microseconds [8]. Comparing the electronic switches, the photogenerated silicon plasma switch of this work achieves the lowest insertion loss, and is orders of magnitude smaller than the silicon plasma switch presented in [19]. 
TABLE I

Performance Comparison of Millimeter-Wave Waveguide Switches

\begin{tabular}{|c|c|c|c|c|c|c|}
\hline Ref. & $\begin{array}{l}\text { Switching } \\
\text { Technology }\end{array}$ & $\begin{array}{c}\text { Frequency Range } \\
\qquad(\mathrm{GHz})\end{array}$ & $\begin{array}{l}\text { Fractional } \\
\text { Bandwidth }\end{array}$ & $\begin{array}{l}\text { Peak Insertion Loss } \\
\qquad(\mathrm{dB})\end{array}$ & $\begin{array}{l}\text { Isolation } \\
(\mathrm{dB})\end{array}$ & $\begin{array}{c}\text { Volume } \\
\text { Normalized to } \lambda^{3 \mathrm{a}}\end{array}$ \\
\hline This Work & Photogenerated Silicon Plasma & $29.2-41.6$ & $35 \%$ & 0.52 & $>25$ & 0.005 \\
\hline [19] & Photogenerated Silicon Plasma & $170-260$ & $42 \%$ & 0.7 & $>45$ & 3.98 \\
\hline [14] & Photogenerated Silicon Plasma in SIW & $23.3-26.5$ & $13 \%$ & 0.7 & $>22$ & 0.017 \\
\hline [4] & PIN Diode ${ }^{b}$ & $60-90$ & $40 \%$ & 2.5 & 20 & 131.8 \\
\hline [41] & PIN Diode in SIW & $20-24$ & $18 \%$ & 1.3 & $>10$ & 0.11 \\
\hline [3] & Electromechanical $^{\mathrm{c}}$ & $26.5-40$ & $41 \%$ & 0.4 & 60 & 211.4 \\
\hline [42] & Mechanical in SIW & $50-75$ & $40 \%$ & 4.5 & $>45$ & 0.096 \\
\hline [7] & RF MEMS & $60-80$ & $29 \%$ & $<0.2$ & $>20$ & 0.002 \\
\hline [8] & RF MEMS & $60-70$ & $15 \%$ & 0.4 & $>30$ & 0.22 \\
\hline [10] & Microfluidic & $25-40$ & $46 \%$ & $<0.5$ & $>22$ & 1.47 \\
\hline
\end{tabular}

${ }^{a}$ In the respective material at lowest band frequency.

${ }^{\mathrm{b}}$ QuinStar Technology, Inc. QSD series.

${ }^{\mathrm{c}}$ QuinStar Technology, Inc. QWZ series.

\section{CONCLUSION}

A photogenerated silicon plasma $\pi$-match waveguide switch using silicon bulk micromachining was presented for operation at millimeter-wave frequencies. A theoretical study of the photoconductivity model along with the design procedure for a $\pi$-match waveguide switch were discussed, with measured results agreeing well with simulation. The reported measured ON-state return loss was below $20 \mathrm{~dB}$ from $30-40 \mathrm{GHz}$, with extracted insertion loss as low as $0.52 \mathrm{~dB}$ at $35 \mathrm{GHz}$, and less than $0.88 \mathrm{~dB}$ across the entire band. The OFF-state isolation was greater than $25 \mathrm{~dB}$ at $35 \mathrm{GHz}$, and showed good variability versus optical excitation energy for application as a variable attenuator. A microfabrication process flow using deep reactive ion etching to form silicon posts within the waveguide channel was presented, allowing for both high accuracy and low cost manufacturing due to economies of scale. The presented results highlight the significant potential of silicon micromachined waveguide switches using optically excited plasma tuning elements for controling millimeter-wave signals in high power and high speed switching applications.

\section{REFERENCES}

[1] U. Shah, T. Reck, H. Frid, C. Jung-Kubiak, G. Chattopadhyay, I. Mehdi, and J. Oberhammer, "A 500-750 GHz RF MEMS waveguide switch," IEEE Transactions on Terahertz Science and Technology, vol. 7, no. 3, pp. 326-334, May 2017.

[2] Logus Microwave Corporation, (accessed on May 26, 2021). [Online]. Available: https://www.logus.com/products/waveguide-switches/

[3] QuinStar Technology Inc., (accessed on May 26, 2021). [Online]. Available: https://quinstar.com/shop/test-equipmentinstruments/electromechanical-waveguide-switches/electromechanicalwaveguide-switches-qwz/

[4] QuinStar Technology Inc., (accessed on May 26, 2021). [Online]. Available: https://quinstar.com/shop/control-products-ferrite/waveguidepin-switches-single-double-throw/waveguide-pin-switches-doublethrow-qss-qsd/

[5] T. Sickel, P. Meyer, and P. W. Van Der Walt, "An in situ tunable diode mounting topology for high-power X-band waveguide switches," IEEE Transactions on Microwave Theory and Techniques, vol. 55, no. 2, pp. 281-285, 2007.
[6] D. Psychogiou, Y. Li, J. Hesselbarth, S. Kuhne, D. Peroulis, C. Hierold, and C. Hafner, "Millimeter-wave phase shifter based on waveguidemounted RF-MEMS," Microwave and Optical Technology Letters, vol. 55, no. 3, pp. 465-468, Mar 2013.

[7] N. Vahabisani and M. Daneshmand, "Monolithic millimeter-wave MEMS waveguide switch," IEEE Transactions on Microwave Theory and Techniques, vol. 63, no. 2, pp. 340-351, Feb 2015.

[8] Z. Baghchehsaraei and J. Oberhammer, "Parameter analysis of millimeter-wave waveguide switch based on a MEMS-reconfigurable surface," IEEE Transactions on Microwave Theory and Techniques, vol. 61, no. 12, pp. 4396-4406, Dec 2013.

[9] L. Pelliccia, F. Cacciamani, P. Farinelli, and R. Sorrentino, "HighQ tunable waveguide filters using ohmic RF MEMS switches," IEEE Transactions on Microwave Theory and Techniques, vol. 63, no. 10, pp. 3381-3390, Oct 2015.

[10] C. H. Chen and D. Peroulis, "RF design, power handling, and hot switching of waveguide water-based absorptive switches," IEEE Transactions on Microwave Theory and Techniques, vol. 57, no. 1, pp. 2038-2046, Aug 2009.

[11] S. Khan, N. Vahabisani, and M. Daneshmand, "A fully 3-D printed waveguide and its application as microfluidically controlled waveguide switch," IEEE Transactions on Components, Packaging and Manufacturing Technology, vol. 7, no. 1, pp. 70-80, Jan 2017.

[12] C. D. Gamlath, D. M. Benton, and M. J. Cryan, "Microwave properties of an inhomogeneous optically illuminated plasma in a microstrip gap," IEEE Transactions on Microwave Theory and Techniques, vol. 63, no. 2, pp. 374-383, Feb 2015.

[13] C. H. Lee, P. S. Mak, and A. P. Defonzo, "Optical Control of MillimeterWave Propagation in Dielectric Waveguides," IEEE Journal of Quantum Electronics, vol. 16, no. 3, pp. 277-288, 1980.

[14] E. Shepeleva, M. Makurin, A. Lukyanov, A. R. Vilenskiy, S. L. Chernyshev, and M. V. Ivashina, "Low-loss K-band Photoconductive Switches in SIW Technology," in Proceedings of the 50th European Microwave Conference, Utrecht, The Netherlands, Jan 2021, pp. 538-541.

[15] A. Fisher, Z. Missen, T. Jones, and D. Peroulis, "A fiber-free DC-7GHz $35 \mathrm{~W}$ integrated semiconductor plasma switch," in IEEE International Microwave Symposium, IMS, Atlanta, GA, Jun 2021, pp. 1-4.

[16] A. W. Pang, S. Bensmida, and M. J. Cryan, "Nonlinearity and Power Handling Characterization of an Optically Reconfigurable Microwave Switch," in IEEE MTT-S International Microwave Symposium Digest, Philadelphia, PA, Jun 2018, pp. 420-422.

[17] E. K. Kowalczuk, C. J. Panagamuwa, and R. D. Seager, "Design and operation influences regarding rise and fall time of a photoconductive microwave switch," in Loughborough Antennas and Propagation Conference, LAPC, Loughborough, UK, Nov 2013, pp. 149-154.

[18] A. W. Pang, C. D. Gamlath, and M. J. Cryan, "An optically controlled coplanar waveguide millimeter-wave switch," IEEE Microwave and Wireless Components Letters, vol. 28, no. 8, pp. 669-671, 2018.

[19] J. Ren, Z. Jiang, P. Fay, J. L. Hesler, C. Y. E. Tong, and L. Liu, "Highperformance WR-4.3 optically controlled variable attenuator with 60 - 
$\mathrm{dB}$ range," IEEE Microwave and Wireless Components Letters, vol. 28, no. 6, pp. 512-514, Jun 2018.

[20] J. Campion, Y. Li, H. Zirath, J. Oberhammer, A. Hassona, Z. S. He, B. Beuerle, A. Gomez-Torrent, U. Shah, S. Vecchiattini, R. Lindman, and T. S. Dahl, "Toward industrial exploitation of THz frequencies: integration of SiGe MMICs in silicon-micromachined waveguide systems," IEEE Transactions on Terahertz Science and Technology, vol. 9, no. 6, pp. 624-636, Nov 2019.

[21] A. Krivovitca, U. Shah, O. Glubokov, and J. Oberhammer, "Micromachined silicon-core substrate-integrated waveguides at 220-330 GHz," IEEE Transactions on Microwave Theory and Techniques, vol. 28, no. 12, pp. 5123-5131, Dec 2020.

[22] A. Kannegulla, M. I. B. Shams, L. Liu, and L.-J. Cheng, "Photo-induced spatial modulation of THz waves: opportunities and limitations," Optics Express, vol. 23, no. 25, pp. 32 098-32 112, Dec 2015.

[23] Ioffe Physical-Technical Institute, (accessed on April 28, 2021). [Online]. Available: http://www.ioffe.ru/index_en.html

[24] S. Sze and K. N. Kwok, Physics of Semiconductor Devices, 3rd ed. Hoboken, New Jersey: John Wiley \& Sons, Inc, 2007.

[25] J. Geist, "Silicon (Si) Revisited (1.1-3.1 eV)," in Handbook of Optical Constants of Solids. San Diego, CA: Academic Press, 1998, pp. 519529.

[26] D. K. Schröder, "Carrier lifetimes in silicon," IEEE Transactions on Electron Devices, vol. 44, no. 1, pp. 160-170, Jan 1997.

[27] S. Selberherr, Analysis and Simulation of Semiconductor Devices. New York: Springer-Verlag Wien, 1984.

[28] C. Hu, Modern Semiconductor Devices for Integrated Circuits. Upper Saddle River: Prentice Hall, 2010.

[29] R. Ulbricht, E. Hendry, J. Shan, T. F. Heinz, and M. Bonn, "Carrier dynamics in semiconductors studied with time-resolved terahertz spectroscopy," Reviews of Modern Physics, vol. 83, no. 2, pp. 543-586, 2011.

[30] E. Episkopou, S. Papantonis, W. J. Otter, and S. Lucyszyn, "Defining material parameters in commercial em solvers for arbitrary metalbased THz structures," IEEE Transactions on Terahertz Science and Technology, vol. 2, no. 5, pp. 513-524, Sep 2012.

[31] G. M. Rebeiz, RF MEMS Theory, Design, and Technology. Hoboken, NJ: John Wiley \& Sons, Inc., 2003.

[32] P. A. Rizzi, Microwave Engineering: Passive Circuits. Englewood Cliffs, NJ: Prentice Hall, 1998.

[33] B. Pan, Y. Li, M. M. Tentzeris, and J. Papapolymerou, "Surface micromachining polymer-core-conductor approach for high-performance millimeter-wave air-cavity filters integration," Ieee Transactions On Microwave Theory And Techniques, vol. 56, no. 4, pp. 959-970, Apr 2008.

[34] Sheaumann Laser Inc., (accessed on April 26, 2021). [Online]. Available: https://www.sheaumann.com/

[35] S. M. Sze, Semiconductor Devices: Physics and Technology, 2nd ed. New York: John Wiley Sons, Inc., 2002.

[36] Wavelength Electronics, (accessed on April 26, 2021). [Online]. Available: https://www.teamwave length.com/download/Datasheets/LD15 CHA.pdf

[37] OZ Optics, (accessed on April 26, 2021). [Online]. Available: https://www.ozoptics.com/ALLNEW_PDF/DTS0095.pdf

[38] K. N. Chen, C. S. Tan, A. Fan, and R. Reif, "Copper bonded layers analysis and effects of copper surface conditions on bonding quality for three-dimensional integration," Journal of Electronic Materials, vol. 34, no. 12, pp. 1464-1467, Dec 2005.

[39] D. M. Pozar, Microwave Engineering, 4th ed. Hoboken, NJ: John Wiley Sons, Inc., 2012.

[40] A. Enders and G. Nimtz, "On superluminal barrier traversal," Journal de Physiques I, vol. 2, no. 9, pp. 1693-1698, Sep 1992.

[41] A. B. Numan, J.-f. Frigon, and J.-j. Laurin, "Single-pole single-throw switch for substrate-integrated waveguide," IEEE Microwave and Wireless Components Letters, vol. 28, no. 3, pp. 221-223, mar 2018.

[42] S. C. Wei, C. H. Yang, Y. C. Chen, T. A. Chen, and C. Y. Chang, "Vand W-band substrate integrated waveguide (SIW) mechanical switch," IEEE Transactions on Microwave Theory and Techniques, vol. 66, no. 6, pp. 3090-3098, jun 2018 